Gielen, S.C., Dekker, J., Francke, A.L., Mistiaen, P., Kroezen, M. The effects of nurse prescribing: a systematic review. International Journal of Nursing Studies: 2014, 51(7), 1048 1061

\begin{tabular}{|l|l|}
\hline $\begin{array}{l}\text { Postprint } \\
\text { Version }\end{array}$ & 1.0 \\
\hline Journal website & http://www.sciencedirect.com/science/article/pii/S0020748913003714 \\
\hline Pubmed link & $\underline{\text { http://www.ncbi.nlm.nih.gov/pubmed/24398118 }}$ \\
\hline DOI & $10.1016 /$ j.jnurstu.2013.12.003 \\
\hline
\end{tabular}

This is a NIVEL certified Post Print, more info at http://www.nivel.eu

\title{
The effects of nurse prescribing: A systematic review.
}

\author{
SANNE C. GIELEN ${ }^{\mathrm{A}, 1}$, JANNEKe DeKKER ${ }^{\mathrm{B}, 1}$, ANNEKe L. FrANCKE ${ }^{\mathrm{C}, \mathrm{D}}$, PATRIEK Mistiaen ${ }^{\mathrm{C}}$, \\ MARIEKE KROEZEN ${ }^{\mathrm{C}, 1, *}$ \\ ${ }^{a}$ Institute of Nursing Studies of HAN University of Applied Sciences, Nijmegen, The \\ Netherlands \\ ${ }^{\mathrm{b}}$ Department of Neonatology of LUMC, Leiden, The Netherlands \\ ${ }^{c}$ NIVEL, Netherlands Institute for Health Services Research, Utrecht, The Netherlands \\ ${ }^{\mathrm{d}}$ Department of Public and Occupational Health, EMGO Institute for Health and Care \\ Research (EMGO+) of VU University Medical Center, Amsterdam, The Netherlands
}

\begin{abstract}
A B S T RA C T
Background: In 2008, we conducted a systematic review on the effects of nurse prescribing using studies with a comparative design. In view of the growing number of countries that are introducing nurse prescribing and the fact that several studies into nurse prescribing have been conducted recently, there is a need for an updated review to reassess the available information on the effects of nurse prescribing when compared to physician prescribing. Objective: To identify, appraise and synthesise the evidence on the effects of nurse prescribing when compared to physician prescribing on the quantity and types of medication prescribed and on patient outcomes. Design: A systematic review. Data sources: In addition to the previous review, which covered the literature up to 2005, 11 literature databases and four websites were searched for relevant studies from January 2006 up to January 2012 without limitations as to language or country. Moreover, full-text copies of all studies included in the previous review were reviewed. Review methods: A three-stage inclusion process, consisting of an initial sifting, checking full-text papers for inclusion criteria and methodological assessment, was performed independently by two reviewers. Data on effects were synthesised using narrative and tabular methods. Results: Thirty-five studies met the inclusion criteria. All but five studies had a high risk of bias. Nurses prescribe in comparable ways to physicians. They prescribe for equal numbers of patients and prescribe comparable types and doses of medicines. Studies comparing the total amount of medication prescribed by nurses and doctors show mixed results. There appear to be few differences between nurses and physicians in patient health outcomes: clinical parameters were the same or better for treatment by nurses, perceived quality of care was similar or better and
\end{abstract}


patients treated by nurses were just as satisfied or more satisfied. Conclusions: The effects of nurse prescribing on medication and patient outcomes seem positive when compared to physician prescribing. However, conclusions must remain tentative due to methodological weaknesses in this body of research. More randomised controlled designs in the field of nurse prescribing are required for definitive conclusions about the effects of nurse prescribing.

What is already known about this topic?

- Over the last two decades, the number of countries in which nurses are legally permitted to prescribe medication has grown considerably.

- In 2008, we conducted a systematic review on the effects of nurse prescribing using studies with a comparative design to compare the effects of nurse prescribing to physician prescribing.

- In view of the growing number of countries that are introducing nurse prescribing and the fact that several studies into nurse prescribing have been conducted recently, there is a need for an updated review.

What this paper adds

- Our updated systematic review suggests that nurses prescribe for a wide range of patients and in comparable ways to physicians.

- Patients were generally more or equally satisfied with the care provided by nurses compared to the traditional care provided by physicians. - Conclusions must remain tentative due to methodolo- gical weaknesses in this body of research. More randomised controlled designs in the field of nurse prescribing are required for definitive conclusions about the effects of nurse prescribing.

\section{INTRODUCTION}

\subsection{Background}

Nurses can legally prescribe medication in quite a number of countries nowadays, including Australia, Canada, Finland, Ireland, New Zealand, Norway, South Africa, Sweden, the Netherlands, the United Kingdom and the United States of America (Aarts and Koppel, 2009; Ball, 2009; Drennan et al., 2009; Kroezen et al., 2011, 2012; Ministry of Health WaS, 2011; Van Ruth et al., 2008). The extension of prescribing rights to nurses has been intro- duced for several reasons. It is expected, among others, that nurse prescribing will contribute to efficient and effective patient care and will improve the quality and continuity of care (Buchan and Calman, 2004; Department of Health, 1999, 2002; Dutch House of Representatives, 2011; Emmerton et al., 2005; Kroezen et al., 2011; Ministry of Health WaS, 2011; Van Ruth et al., 2008). Moreover, nurse prescribing offers the potential to make better use of nurses' professional skills, increase nurses' autonomy and yield time savings for medical practitioners and patients (Bradley and Nolan, 2007; Buchan and Calman, 2004; Department of Health, 1999; Kroezen et al., 2011; Raad voor, 2002).

Even though the term 'nurse prescribing' suffices as a descriptor, the actual practice it refers to varies consider- ably, both within countries and internationally (Kroezen 
et al., 2011, 2012; Jones, 2009). Nonetheless, three general models of nurse prescribing are usually distinguished in the literature, viz. independent prescribing, supplemen- tary prescribing and prescribing based on patient group directions (see Box 1). This review will adhere to this general classification.

In 2008, the Netherlands Institute for Health Services Research (NIVEL) conducted a systematic literature review of the effects of nurse prescribing using studies with a comparative design (Van Ruth et al., 2008). In this review we concluded that overall, the effects of nurse prescribing appeared to be positive. However, of the 23 studies that were included in the review, all but two had a high or moderate risk of bias, based on the EPOC criteria (Cochrane Effective Practice and Organisation of Care Review Group, 2002). The present systematic review is an update of this earlier review (Van Ruth et al., 2008). Since our previous review was published, nurse prescribing has been introduced in two more countries, viz. Finland and the Netherlands (Ministry of Health WaS, 2011; Pharmaceutisch Weekblad, 2011).

\section{[Box 1]}

Moreover, quite a number of studies and evaluations from other countries have appeared. Nurse prescribing has been in place by now for a substantial number of years in some countries, such as Ireland and the UK, increasing the opportunity for more in-depth research and publications. In view of the growing number of countries that are introducing nurse prescribing and the fact that several studies into nurse prescribing have been conducted recently, there is a need for an updated review to reassess the available information on the effects of nurse prescribing.

While several reviews have been conducted into the legal and/or educational conditions under which nurse prescribing has been implemented in different countries (Banning, 2004; Kroezen et al., 2011; Ryan et al., 1999), and other nonsystematic reviews have addressed the advan- tages of nurse prescribing in terms of access and delivery of care and nurses' knowledge and skills (An Bord, 2005; Creedon et al., 2009; Harris et al., 2004), few reviews have examined the effects of nurse prescribing on medication and patient outcomes. These are important outcome measures though, firstly because nurse prescribing is often introduced to improve the quality of care, of which medication and patient outcomes are important measures. In the second place they are important because questions have been raised about the adequacy of nurses' educa- tional programmes and whether nurses have the competence to prescribe medicines (Avery and Pringle, 2005; Banning, 2004; Crown and Miller, 2005; Horton, 2002; Nilsson, 1994; Siriwardena, 2006; Wilhelmsson and Foldevi, 2003).

As said, in 2008 we conducted a systematic review of the effects of nurse prescribing on medication and patient outcomes using studies with a comparative design. Three other reviews also studied the effects of nurse prescribing, but these lacked a comparative design (Bhanbhro et al., 2011; Latter and Courtenay, 2004; O’Connell et al., 2009; Van Ruth et al., 2008). Latter and Courtenay (2004) found that nurse prescribing has generally been evaluated positively (Latter and Courte- nay, 2004). However, their review lacked a systematic approach as well as a comparative design. O’Connell et al. (2009) reported advantages of nurse prescribing for both patients and nurse prescribers, but concluded that further research, preferably randomised controlled trials (RCTs), would be useful to determine the benefits of nurse 
prescribing versus doctor prescribing (O’Connell et al., 2009). Bhanbhro et al. (2011) conducted a systematic literature review on the contribution of prescribing in primary care by nurses, indicating that nurse prescribing effectively improves patients' condition and provides a better level of care. However, many of the studies included in this review had design weaknesses and limitations, and only two presented comparative data about general practitioners. Hence, these reviews into the effects of nurse prescribing all lacked a comparative design to compare the effects of nurse prescribing to physician prescribing. However, a comparison with the traditional approach of prescribing by medical professionals is a necessary prerequisite in order to properly assess the value of nurse prescribing.

\subsection{Aim and research questions}

The aim of this updated review was to identify, appraise and synthesise the evidence presented in the literature on the effectiveness of nurse prescribing compared to physician prescribing. We looked for evidence about the effects of nurse prescribing on the quantity and types of medication and on patient outcomes. The following research questions were addressed:

1. What are the effects of nurse prescribing on the quantity and types of medication being prescribed?

2. What are the effects of nurse prescribing on patient outcomes?

\section{MethodS}

A more stringent update of the systematic literature review by Van Ruth et al. (Van Ruth et al., 2008) was conducted, working in accordance with the steps in the Preferred Reporting Items for Systematic Reviews and Meta-Analyses (PRISMA) Statement (Moher et al., 2009) and the Cochrane Handbook for Systematic Reviews (Higgins and Green, 2011). This update differs from the previous review in that it does not include qualitative study designs. Furthermore, in contrast to the earlier review, we do not address the effects of nurse prescribing on physician and nurse outcomes and characteristics of the health-care system.

\subsection{Search strategy}

To identify all relevant studies up to January 2012, the following 11 literature databases and four websites were searched: BioMed Central, CINAHL, Cochrane Database of Systematic Reviews, Current Controlled Trials, Embase, INVERT (Dutch nursing literature index), NIVEL catalo- gue, PiCarta (Dutch library system), PubMed, Science Citation Index and the Virginia Henderson International Nursing Library, and the website of the UK Department of Health (www.doh.gov.uk), the website of the World Health Organisation (www.who.org), a website for health professionals (www.escriber.com) and Google Scholar (www.scholar.google.com). All databases and websites were searched from January 2006 up to January 2012 without limits as to country or language. The search was highly sensitive. The following search strategy was used for PubMed: ("Nurse prescribing') OR (Nurs* [tiab] AND Prescri* [tiab]) OR (Nurs* AND prescriptions, drug [MeSH]), and suitable search strate- gies were developed for the other databases using adaptations 
Gielen, S.C., Dekker, J., Francke, A.L., Mistiaen, P., Kroezen, M. The effects of nurse prescribing: a systematic review. International Journal of Nursing Studies: 2014, 51(7), 1048 1061

of the PubMed search. All the detailed search strategies can be found in the additional file 1 , 'Search strategies'. The hits from all the searches were entered into Reference Manager@; duplicates were eliminated in this program and then the inclusion process was carried out.

\subsection{INCLUSION AND EXCLUSION CRITERIA}

Our inclusion and exclusion criteria were structured according to the PICO (Patients, Intervention, Comparison, Outcome) method.

\subsubsection{Patients}

All patient groups were included. There was no restriction in terms of age.

\subsubsection{Intervention}

Studies were included that addressed the nurse prescribing of medicines as defined in one of the three general models of nurse prescribing, i.e. independent prescribing, supplementary prescribing or prescribing by patient group directions. Studies that only concerned nurse prescribing based on group protocols for child vaccination or travel vaccination were excluded, as these severely limit nurses' prescribing rights.

\subsubsection{Comparison}

Studies needed to have a comparative design in which nurse prescribing was compared to physician prescribing in order to be included in the review. Studies comparing nurse prescribing to prescribing by other non-medical prescribers (e.g. pharmacists) were excluded.

\subsubsection{Outcome}

All studies were included that reported on the effects of nurse prescribing on the quantity and/or types of medica- tion prescribed and/or on patient outcomes. We did not apply a strict definition of patient outcome measures. All studies with outcome measures that said something about the effects of nurse prescribing on patient outcomes were included (e.g. patients' clinical parameters, satisfaction with care or number of patient visits to the prescriber).

\subsubsection{Type of study}

Only primary research studies with a quantitative design were included. Studies with a qualitative design and publications that were not primary research studies, i.e. letters, abstracts, reviews and editorials, were excluded.

A three-stage inclusion process was applied. Initially, a 10\% sample of all nonduplicate references found in the literature search was studied independently by two reviewers (JD and SG), looking at the title and abstract; references were included in the study if they met the above criteria. It was stipulated beforehand that if there was substantial agreement between the two reviewers for this $10 \%$ sample, the remaining $90 \%$ of the sample would be divided between them. In accordance with the prevailing cut-off points in the literature, a Kappa value between 0.60 and 0.80 was considered an indication of good/substantial agreement (Landis and Koch, 1977). If 
the title and abstract provided insufficient information to determine relevance, full paper copies of the articles were ordered and these articles were included in the second selection round.

In the second stage, both reviewers independently examined all full paper copies of the articles selected in the first stage, in order to determine whether they fulfilled the inclusion criteria. Disagreements were either resolved by discussion or the final decision was made by a third reviewer.

Finally, full-text copies of all 23 studies included in the previous review (Van Ruth et al., 2008) were checked by both reviewers to see whether they fulfilled the stricter inclusion criteria of this review.

\subsection{Quality assessment}

The methodological quality of the studies included was assessed independently by two reviewers (JD and SG) using the quality criteria of the Cochrane Effective Practice and Organisation of Care (EPOC) Review Group (Cochrane Effective Practice and Organisation of Care Review Group, 2002). Differences were resolved by consensus. The EPOC quality criteria checklist includes seven criteria for randomised controlled trials and controlled clinical trials (CCTs), seven criteria for controlled before-and-after studies (CBAs) and seven criteria for interrupted time series (ITS). The EPOC criteria used to assess RCTs and CCTs are: concealment of allocation, follow-up of professionals, follow-up of patients or episodes of care, blind assessment of primary outcome(s), baseline measurement, reliable primary outcome measure(s) and protection against contamination.

We assigned an overall quality rating (high, moderate or low risk of bias) to each study. A study was judged as having a low risk of bias if it met all seven criteria, a moderate risk of bias if it met four, five or six criteria, and a high risk of bias if it met three criteria or fewer (Cochrane Effective Practice and Organisation of Care Review Group,

2002). The EPOC criteria were not developed to assess the methodological quality of study designs other than RCTs, CCTs, CBAs and ITS. Other designs, such as preexperi- mental post-test only designs, were judged as having a high risk of bias, as these generally have low evidence strength when studying the effects of interventions.

\subsection{Data analysis and synthesis}

The first two authors (JD and SG) extracted data from the publications included and entered the data onto digital structured data-extraction forms; the last author (MK) checked the extracted data. Disagreements were resolved by discussion between the review authors. Data were extracted about the country, model of nurse prescribing, prescribers, patients, number of medicines prescribed, types of medicines prescribed and various patient out- comes. All data extracted from the studies were based on the results sections and not on the study conclusions. Outcomes were classified according to the research questions and grouped together into effects on the medication prescribed and effects on patient outcomes. 
Gielen, S.C., Dekker, J., Francke, A.L., Mistiaen, P., Kroezen, M. The effects of nurse prescribing: a systematic review. International Journal of Nursing Studies: 2014, 51(7), 1048 1061

\subsubsection{Pooling}

The option of pooling published effect sizes was considered if studies reported similar outcomes, presented raw data and reported outcomes that were either all continuous or all dichotomous. Standardised mean differ- ences and a random effects model were used for continuous outcomes, while relative risks and a random effects model were used for dichotomous outcomes.

Confidence intervals were set at 95\% (Higgins and Green, 2005). The decision to pool studies was based on their clinical homogeneity, defined as similarity in the care setting and in the type of illness affecting the patients included in the study. Furthermore, the results of pooling are only reported if the pooled studies show acceptable statistical homogeneity. Studies were considered to be statistically homogenous if the chi-square test value was less than the degrees of freedom, the $\mathrm{P}$ value was above 0.1 and the inconsistency test I2 was less than 50\% (Clarke and Oxman, 2003; Higgins and Green, 2005).

\subsubsection{Subgroup analysis}

If data permitted, we planned to conduct subgroup analyses for different countries, nurse prescribers and models of nurse prescribing. However, due to substantial statistical heterogeneity between studies, this was only possible for countries.

\subsubsection{Sensitivity analysis}

The intention was to conduct a sensitivity analysis on the basis of study quality. However, as almost all studies included had a high risk of bias, no sensitivity analysis was performed.

\section{RESULTS}

\subsection{Search and inclusion results}

After duplicates had been removed, the searches in the different databases resulted in an initial set of 6588 references of potential interest. Initial sifting based on title and abstract reduced this set to 593 references. As said, a 10\% sample of all references was initially studied independently by two reviewers (JD and SG), looking at the title and abstract. These reviewers had good/moderate agreement (Cohen's Kappa = 0.76), and the remaining $90 \%$ of the sample was therefore divided between them. Full- text copies were ordered of the resulting set of 593 studies for the second stage of the inclusion process; 578 copies were actually obtained.

Two reviewers (JD and SG) independently performed the second selection phase and 14 studies were deemed eligible for inclusion. Additionally, the two reviewers checked all studies included in the previous review for eligibility in this review and included 21 of them. Finally, 35 studies were selected for the next stage of the review, for data-extraction and analysis. Fig. 1 shows the flow diagram of the inclusion process. 


\subsection{Methodological quality of the final 35 studies}

Only 10 of the 35 studies included were randomised controlled trials (Einhorn and Trias, 1978; Houweling et al., 2005a,b,c, 2009; Kuethe et al., 2011; Shum et al., 2000; Spitzer et al., 1974; Tobe et al., 2006; Venning et al., 2000), and one was a Controlled Clinical Trial (Kinnersley et al., 2000). The methodological quality of these 11 studies using a randomised or non-randomised controlled design was assessed using the EPOC criteria for RCTs and CCTs.

Three studies had a low risk of bias (Houweling et al., 2009; Kuethe et al., 2011; Tobe et al., 2006), two studies had a moderate risk of bias (Houweling et al., 2005a,c) and the remaining RCTs and the CCT had a high risk of bias (Einhorn and Trias, 1978; Houweling et al., 2005b; Kinnersley et al., 2000; Shum et al., 2000; Spitzer et al., 1974; Venning et al., 2000). The remaining 24 studies either had a pretest post-test design without a compar- ison group or a pre-experimental post-test only design. As these designs generally have low evidence strength when studying the effects of interventions, they were all rated as having a high risk of bias.

\subsection{Characteristics of the final 35 studies}

\subsubsection{Date and geographical focus of studies}

The publication years of the selected studies varied from 1974 to 2011. Thirteen of the 35 studies were conducted in the USA, twelve in the UK, five in the Netherlands, two in Canada, two in Norway and one in Colombia.

\subsubsection{Nurse prescribing models studied}

Twenty-two of the 35 studies involved independent nurse prescribing, two studies involved supplementary nurse prescribing, five studies described a mix of independent and supplementary prescribing, and six studies looked at prescribing based on group directions.

\subsubsection{Care setting}

Twenty-three studies were conducted in primary care (Batey and Holland, 1985; Butler et al., 2001; Cipher et al., 2006; Cox and Jones, 2000; Davis and Drennan, 2007; Einhorn and Trias, 1978; Ferguson et al., 1998; Fletcher et al., 2011; Hansen and Skjeldestad, 2007; Hooker and Cipher, 2005; Houweling et al., 2005c; Kinnersley et al., 2000; Ladd, 2005; Pritchard and Kendrick, 2001; Roumie et al., 2005; Running et al., 2006; Sandø et al., 2010; Shum et al., 2000; Spitzer et al., 1974; Tobe et al., 2006; Undeland et al., 2010; Venning et al., 2000; Williams et al., 2009), eleven studies were conducted in secondary care (Hou- weling et al., 2005a,b, 2009; Feldman et al., 2003; Fisher and Vaughan-Cole, 2003; Foreman and Morton, 2011; Gambino et al., 2009; Jacobs, 2005; James, 2004; Jones et al., 2011; Mallett et al., 1997) and one study was conducted in both primary and secondary care (Kuethe et al., 2011). 
Gielen, S.C., Dekker, J., Francke, A.L., Mistiaen, P., Kroezen, M. The effects of nurse prescribing: a systematic review. International Journal of Nursing Studies: 2014, 51(7), 1048 1061

\section{[FIGURE 1].}

\subsubsection{Patients of interest in the studies}

Some of the studies involved nurse prescribing for several or mixed patient populations, while others were restricted to nurse prescribing for specified patient groups only (e.g. patients with diabetes, mental health patients or patients with acute minor illnesses). Eleven studies involved nurse prescribing for various groups of patients (Batey and Holland, 1985; Ferguson et al., 1998; Hooker and Cipher, 2005; Kinnersley et al., 2000; Kuethe et al., 2011; Roumie et al., 2005; Running et al., 2006; Shum et al., 2000; Spitzer et al., 1974; Venning et al., 2000; Williams et al., 2009). Seven studies focused on nurse prescribing for patients with diabetes (Fletcher et al., 2011; Houweling et al., 2005a,b,c, 2006; James, 2004; Tobe et al., 2006), four studies studied nurse prescribing for (cardiology) patients with hypertension (Fletcher et al., 2011; Gambino et al., 2009; Jones et al., 2011; Tobe et al., 2006), four studies were carried out in the field of mental health care (Feldman et al., 2003; Fisher and Vaughan-Cole, 2003; Foreman and Morton, 2011; Jacobs, 2005), three studies included patients with sore throats or upper respiratory throat infections (Butler et al., 2001; Cox and Jones, 2000; Ladd, 2005), three studies involved nurses prescribing birth control pills (Einhorn and Trias, 1978; Hansen and Skjeldestad, 2007; Sandø et al., 2010), one study included children with moderate asthma (Kuethe et al., 2011), one study focused on nurse prescribing for the medical management of constipation (Davis and Drennan, 2007), one study focused on patients with acute minor illnesses (Pritchard and Kendrick, 2001), one study included all patient encounters resulting in a diagnosis of (streptococcal) pharyngitis or sore throat (Undeland et al., 2010), and finally there was one study in a radiotherapy and oncology department that involved patients with diagnoses that included acute radiation toxicity causing proctitis from pelvic radiotherapy and erythema of the scalp due to cranial irradiation (Mallett et al., 1997).

\subsection{Effects on medication prescribed}

All results discussed in the following sections were statistically significant at $\mathrm{P}<$ 0.05 unless otherwise indicated.

\section{[TABLE 1].}

\subsubsection{Total amount of medication prescribed}

Studies comparing the total amount of medication prescribed by nurses and doctors show mixed results. As most studies found divergent results for different types of medicines, it is difficult to determine whether nurses prescribe less, more or the same amount of medication compared to doctors (see Table 1).

Only three of the nine studies reporting on the total amount of medication prescribed found unambiguous results (Ferguson et al., 1998; Houweling et al., 2009; Sandø et al., 2010). Ferguson et al. (1998) concluded that the increase in the volume of prescribing following the introduction of nurse prescribing in the UK was similar to the national increase in the volume of prescribing for the same period. Sandø et al. 
(2010) found that GPs and nurses prescribed equal numbers of initial prescriptions of the birth control pill. Houweling et al. (2009) found that nurses prescribed less cholesterol-lowering medication than medical specialists. Other studies found mixed results depending on therapy type and/or the type of medication being prescribed. While Houweling et al. (2005a) found that specialist nurses and medical specialists prescribed the same amount of glucose and blood pressure lowering medica- tion, specialised nurses prescribed less cholesterol-low- ering medication (Houweling et al., 2005b). Fletcher et al. (2011) concluded that there were no significant differences between nurses and physicians in the prescription of hypoglycaemic medication for patients with hypertension and/or diabetes, but patients were less likely to be prescribed cardiovascular agents if they had a nurse as their primary care provider. Fisher and Vaughan-Cole (2003) found that psychiatrists and advanced practice registered nurses (APRNs) prescribed similar overall quantities of medication except for benzodiazepines, where prescriptions by psychiatrists were more than double the volume prescribed by APRNs. However, the significance of this finding remains unclear (Fisher and Vaughan-Cole, 2003).

Running et al. (2006) found conflicting results. On the one hand, nurses prescribed more over-the-counter (OTC) decongestants for patients with bronchitis than physicians did, but on the other hand, they prescribed fewer bronchodilators (Running et al., 2006). In 2005, Jacobs also found conflicting results, without reporting signifi- cance levels though (Jacobs, 2005). This study found that nurses prescribed fewer mood stabilizers, fewer secondary anti-depressants and less newage antipsychotic medica- tion than psychiatrists. However, where split therapy was concerned, i.e. where patients see a clinician for psy- chotherapy and another healthcare professional for medication management, the prescription of benzodiazepine anti-anxiety agents was slightly higher for patients who received their prescriptions from psychiatric nurses (20\%) than for patients who received their prescriptions from psychiatrists (15\%). For other types of therapies, prescriptions of mood stabilisers and secondary anti- depressants by psychiatric nurses were similar to those by psychiatrists (Jacobs, 2005). Finally, Cipher et al. (2006) found conflicting results in their study as well, but these stemmed from a different source, namely geographical area. In metropolitan areas, there was no difference in the average volume of medication prescribed between nurses and physicians. In nonmetropolitan areas however, the average number of prescriptions was greater for nurses (Cipher et al., 2006).

\subsubsection{Number of patients prescribed medication}

Eleven out of fifteen studies on the number of patients prescribed medication report that the number of patients for whom a nurse prescribes medication is similar to the number of patients for whom a physician prescribes medication. Two studies show nurses prescribing medica- tion for a higher percentage of patients than physicians do and one study found nurses prescribing for a lower number of patients (see Table 2). Most studies found no differences between nurses and doctors regarding the number of patients who were prescribed medication, including prescriptions for antibiotics, anti-depressants, inhaled corticosteroids and medication for hypertension (Cipher et al., 2006; Cox and Jones, 2000; Feldman et al., 2003; Jones et al., 2011; Kinnersley 
Gielen, S.C., Dekker, J., Francke, A.L., Mistiaen, P., Kroezen, M. The effects of nurse prescribing: a systematic review. International Journal of Nursing Studies: 2014, 51(7), 1048 1061

et al., 2000; Kuethe et al., 2011; Ladd, 2005; Pritchard and Kendrick, 2001; Shum et al., 2000; Venning et al., 2000).

\section{[TABLE 2][FIGURE 2].}

Two studies conducted in primary care found that nurses prescribed medication for fewer patients than physicians did (Batey and Holland, 1985; Butler et al., 2001). However, Batey and Holland (1985) do not report whether this difference is statistically significant. Hooker and Cipher (2005) found no difference in the overall number of patients receiving prescriptions, but when rural areas alone were taken into consideration, they found that nurse practitioners prescribed medication for significantly more patients than physicians did. Three studies conducted in primary care also found that nurses prescribed medication for more patients, but Hansen and Skjeldestad (2007) do not report significance levels (Hansen and Skjeldestad, 2007; Hooker and Cipher, 2005; Roumie et al., 2005).

The pooling of studies that reported outcomes on the number of patients being prescribed medication was considered for six studies that all reported raw dichotomous data and were conducted in a primary care setting with various patients. However, it was decided that pooling these studies was not justified due to substantial statistical heterogeneity. But a subgroup analysis based on pooling the country data was possible: four studies from the UK taken together showed no difference in the number of patients being prescribed medication by nurses as compared with GPs (Fig. 2) (Kinnersley et al., 2000; Pritchard and Kendrick, 2001; Shum et al., 2000; Venning et al., 2000).

\section{[TABLE 3].}

\subsubsection{Number of medicines prescribed per patient visit}

Four studies reported on the number of medicines prescribed per patient visit. Two studies concluded that the mean number of medicines prescribed per patient visit was similar for physicians and nurse practitioners (Cipher et al., 2006; Hooker and Cipher, 2005). Jones et al. (2011) found no difference between nurses and doctors in the number of medicines prescribed per patient, while another study found that the average number of medicines used by patients per medicine visit was 1.33 for nurses and 1.87 for physicians, but the statistical significance of this finding was not reported (Batey and Holland, 1985).

\subsubsection{Type of medication and dose prescribed}

There were few overall differences between nurses and doctors in the type and dose of medication prescribed. Jones et al. (2011) reported no statistically significant differences between nurse and doctor prescribers in the types of items prescribed per patient. Kuethe et al. (2011) found that the corrected daily dose of inhaled corticosteroids prescribed for children with asthma was the same for specialised asthma nurses as for GPs and paediatricians. Running et al. (2006) reported that the most common pharmacothera- peutic treatments prescribed by nurses and physicians for patients with musculoskeletal injuries and back pain were non-steroidal anti- 
inflammatory drugs and muscle relax- ants. Nurses appeared to use more nonpharmacological interventions in addition, but these differences were not statistically significant (Running et al., 2006). In mental health care, both psychiatric nurses and psychiatrists primarily prescribed SSRI anti-depressant medicines, but psychiatrists prescribed more other types of anti-depressant medication as well (Feldman et al., 2003; Fisher and Vaughan-Cole, 2003). Finally, the study by Einhorn and Trias (1978) on contraceptives notes that while the number of patients receiving intrauterine devices (IUDs) on a second visit was similar for nurses and physicians, nurses were initially more likely to keep clients on conventional methods such as contraceptive foam and condoms.

Sandø et al. (2010) found a difference in prescribing practices between nurses and GPs, and noted that GPs were more likely than nurses to prescribe a birth control pill of the third generation. Davis and Drennan (2007) likewise concluded that prescribing patterns differed between nurses and GPs, as nearly three-quarters of the prescriptions by nurses for constipation favoured items from the osmotic class of laxatives, compared to only 36\% of GP prescriptions.

\subsection{Effects on patient outcomes}

\subsubsection{Clinical outcomes}

Most of the 13 studies reporting on clinical outcomes found no differences between nurse prescribing and physician prescribing in this regard (see Table 3). There were no significant differences found between patients receiving prescriptions from a nurse and those receiving prescriptions from a physician in HbA1c, blood pressure and creatinine level (Fletcher et al., 2011); in airway responsiveness, asthma control and number of severe exacerbations (Kuethe et al., 2011); in systolic blood pressure, urine albumin status and incidence of adverse events (Tobe et al., 2006); in the number of sore throats that had settled (Cox and Jones, 2000); in the resolution of symptoms and concerns (Kinnersley et al., 2000); in patients' rating of their health status or in terms of clinical improvement after 2 weeks (Shum et al., 2000); in health status outcome (Venning et al., 2000); in the physical status level, emotional and social function, and crude death rates (Spitzer et al., 1974); and in pregnancy rates, method continuation and side effects for contraceptive services (Einhorn and Trias, 1978). Moreover, Houweling et al. conducted several studies in the field of diabetes care and found no significant differences in outcomes for HbA1c, blood pressure, total cholesterol, cholesterol/HDL ratio, lipid profile, quitting smoking rates, percentage of patients within the target values for body mass index (BMI) and quality of life and diabetes-related symptoms (Houweling et al., 2005a,c, 2009).

\section{[TABLE 4].}

Some differences, however, were also reported. Cox and Jones (2000) found that patients' perception of being back to normal health and the median number of days for sore throats to settle were more favourable for nurses than GPs. In another study, patients with hypertension and diabetes receiving prescriptions from nurses had a 
significantly larger drop in diastolic blood pressure than patients receiving nivel prescriptions from physicians (Tobe et al., 2006). Houweling et al. found divergent results for cholesterol/HDL ratios in diabetes patients: in one study, the cholesterol/HDL ratio improved more for patients being treated by a medical specialist (Houweling et al., 2005a), while in another study it improved more for patients being treated by a nurse specialised in diabetes (Houweling et al., 2009).

\subsubsection{Satisfaction with care}

Patients were generally more satisfied or equally satisfied with the care provided by a nurse compared to traditional care provided by a physician. Only one study found that patients treated by a nurse were less satisfied with the care provided than patients cared for by a physician (see Table 4).

Seven studies found that patients were more satisfied with care received from nurses than with care received from GPs or physicians (Gambino et al., 2009; Houweling et al., 2005a,c, 2009; Jones et al., 2011; Shum et al., 2000; Venning et al., 2000).

Moreover, Kinnersley et al. (2000) found that children were more satisfied with care received from nurses, but adult patients did not have a preference. Four other studies found that patients' satisfaction with the care received was similar for nurses and doctors (Cox and Jones, 2000; Foreman and Morton, 2011; Pritchard and Kendrick, 2001; Spitzer et al., 1974). Williams et al. (2009), however, found that patients served by a nurse practi- tioner reported lower levels of satisfaction than patients served by a psychiatrist. However, the roles of the two categories of prescribers in this study were not comparable, so no substantive conclusions can be drawn from these findings (Williams et al., 2009).

\subsubsection{Quality of care}

Two studies concluded that the quality of care provided by nurses was similar to or better than the quality of care provided by GPs. Spitzer et al. (1974) found no significant differences between nurses and GPs in the quality of care, measured on the basis of 10 indicators of care management that were developed by a group of physicians, while Houweling et al. (2005c) showed that all process indicators for the quality of care were higher for nurses than for physicians, except for two indicators for which there was no difference.

\subsubsection{Consultation time}

Six of the eight studies reporting on consultation times found that nurses generally spent more time with patients than doctors, while the remaining two studies found no difference. In the USA, two studies reported that psychia- tric nurses in secondary mental health care spent more time with patients than psychiatrists during medication visits (Fisher and Vaughan-Cole, 2003; Jacobs, 2005), although Jacobs (2005) does not report the statistical significance of this finding. Three studies concluded that nurses had longer consultation times than GPs in general practice in the UK (Kinnersley et al., 2000; Shum et al., 
2000; Venning et al., 2000), and a study in primary diabetes health care in the Netherlands also found that the total duration of the consultations per patient was higher for the practice nurse than for the physicians (Houweling et al., 2005c). However, in the case of secondary diabetes health care, no differences were found in the total duration of all consultations (Houweling et al., 2005b, 2009).

\subsubsection{Provision of information}

Five studies reported that nurses gave more or the same amount of information to patients as doctors. Nurses were found to give more advice about home remedies for sore throats than GPs (Cox and Jones, 2000), and more advice on self-medication and general self-management (Shum et al., 2000). Sandø et al. (2010) reported that significantly more nurses than physicians said that they informed patients about contraceptive security and the risk of arterial thromboembolic disease, and offered follow-up when prescribing OCs for first time. In the study by Kinnersley et al. (2000), patients managed by nurse practitioners (NPs) reported receiving significantly more information about their illnesses. Finally, Running et al. (2006) concluded that NPs and physicians gave similar amounts of smoking cessation information to patients.

\subsubsection{Investigations}

One study in primary care found that nurse practi- tioners were more likely to order tests and investigations than GPs (Venning et al., 2000), while another study found that GPs initiated more investigations for acute minor illnesses compared to practice nurses (Pritchard and Kendrick, 2001). Kinnersley et al. (2000) found no difference between nurse practitioners and GPs in the number of investigations ordered.

\subsubsection{Referrals}

Three studies in primary care reported no differences between nurses and GPs in the number of referrals to secondary care (Kinnersley et al., 2000; Pritchard and Kendrick, 2001; Venning et al., 2000). Two studies of patients with diabetes in secondary care, however, found that patients cared for by specialised nurses were more likely to be referred back to their GP to continue their treatment in the GP practice compared to patients cared for by medical specialists (Houweling et al., 2005a, 2009).

\subsubsection{Follow-up consultations}

In general, patients cared for by nurses make more return visits than patients cared for by doctors. Venning et al. (2000) found that patients treated by nurses were more likely to make return visits to the clinic than patients cared for by GPs. A study of contraceptive services showed that nurses have significantly more scheduled revisits (Einhorn and Trias, 1978), while Kuethe et al. (2011) reported that children cared for by nurses had more regular follow-up visits up than children cared for by GPs or paediatricians. Fletcher et al. (2011) showed that the mean number of primary and specialty care visits did not vary by care provider type, but the average number of 
psychiatric care outpatient visits was significantly lower for nurse practitioners' patients compared with physicians' patients.

Four studies in primary care found no difference between patients cared for by nurses and patients cared for by GPs in the number of follow-up consultations (Butler et al., 2001; Cox and Jones, 2000; Kinnersley et al., 2000; Pritchard and Kendrick, 2001). Only two of the eight studies reporting on follow-up consultations could be pooled with regard to the number of follow-up consulta- tions (Butler et al., 2001; Cox and Jones, 2000). The studies show no significant effect when taken separately, but when pooled, they show that nurses have slightly more follow-up consultations than GPs. However, the rate of follow-up consultations is low (between 6\% and 10\%) for both GPs and nurses, and the effect size found is small (1.68, confidence level 1.04-2.73).

\subsubsection{Medication adherence}

Only one study reported on medication adherence; it found no significant difference between treatment by a psychiatrist and treatment by an advanced practice registered nurse (Jacobs, 2005).

\subsubsection{Patient enablement}

Two studies report that patient enablement, i.e. the extent to which patients understand their illness and are able to cope with it, is similar for nurse practitioners and GPs (Pritchard and Kendrick, 2001; Venning et al., 2000).

\section{DISCUSSION}

This review has identified and mapped quantitative studies exploring the effects of nurse prescribing on medication and patient outcomes. Our results support the findings of the previous review (Van Ruth et al., 2008). Our findings suggest that nurses prescribe for a wide range of patients and in comparable ways to physicians. Overall, nurses appear to prescribe for just as many patients as physicians do, nurses prescribe comparable numbers of medicines per patient visit and there appear to be few differences between nurses and physicians in the type and dose of medication prescribed and in clinical outcomes. Studies comparing the total number of medicines pre- scribed by nurses and doctors show mixed results though, depending on therapy type and the type of medication being prescribed. Patients were generally more or equally satisfied with the care provided by nurses compared to the traditional care provided by physicians. Moreover, nurses generally appear to spend more time with patients than physicians do and to give more or the same amount of information to patients. Results concerning differences in the number of investigations by nurses and physicians are mixed. While there appear to be no differences between nurses and physicians in referrals to secondary care, patients cared for by nurses seem to make more return visits than patients cared for by physicians. Based on these results, it appears that nurse prescribing is of similar quality to physician prescribing, and worries about whether nurses have the competence to prescribe appear to be unfounded. 
Gielen, S.C., Dekker, J., Francke, A.L., Mistiaen, P., Kroezen, M. The effects of nurse prescribing: a systematic review. International Journal of Nursing Studies: 2014, 51(7), 1048 1061

\subsection{Strengths and weaknesses of the review}

The main strength of this review is that it reviews the effects of nurse prescribing on medication and patient outcomes when compared to physician prescribing. The strength of the studies included stems from their real- world setting, with clinically typical, routinely managed patients, and the direct examination of clinically relevant outcomes. Moreover, the datasets of most studies were sufficiently large to explore the phenomenon of interest in this systematic review.

While our findings suggest that nurses prescribe in comparable ways to physicians, the findings should be understood in the context of some limitations. Due to methodological limitations in the studies included, conclusions about the effects of nurse prescribing on medication and patient outcomes remain tentative. We included 35 studies, 24 of which were of low methodological quality owing to their study design, i.e. they were not RCTs or CCTs. Of the eleven RCTs and CCTs included in the review, three had a low risk of bias, two had a moderate risk of bias and six had a high risk of bias. Therefore, only tentative conclusions can be drawn about the effects of nurse prescribing on medication and patient outcomes. Yet it should be noted that the overall general findings as outlined above, indicating that nurses prescribe in similar ways to doctors, are in line with the findings of the five studies that had a moderate to high methodological quality.

Furthermore, our results should be interpreted with caution since a number of other factors may have influenced our results. After all, nurse prescribing is embedded in other tasks such as consultation, diagnosis and treatment. It is difficult to distinguish the effects of nurse prescribing from these other tasks and determine, for example, whether patients are more satisfied with nurse prescribing because of their prescribing practices or because nurses have more time for patients. One possible solution to this problem would be to further elucidate the factors that lead to greater patient satisfaction (Horrocks et al., 2002).

Finally, it should be noted that comparisons between nurses and physicians in the quantity and type of medication prescribed cannot be directly linked to clinical outcomes or effects on patients. Where nurses and physicians prescribe in similar ways, such as the prescription of antibiotics for patients with upper respiratory tract infections, this is usually considered a good thing. However, the prescription of antibiotics is not appropriate for viral upper respiratory tract infections and hence both nurses and physicians, although prescribing in similar ways, are exhibiting suboptimal prescription behaviour in that case (Ladd, 2005). In general, however, nurses appear to prescribe clinically appropriate medication (Black, 2012; Latter et al., 2005; Mahoney, 1994). Moreover, while doctors and nurses within one country will usually prescribe from the same national protocols or guidelines, it is possible that where professionally tailored protocols or guidelines have been developed, comparisons between doctors and nurses in quantity of medicines may be hampered by differences in directions in the protocols and guidelines that are used.

\subsection{Directions for future research}

Future research should preferably employ a randomised controlled design in order to determine the effects of nurse prescribing when compared to physician prescribing on the quantity and types of medication prescribed and on patient outcomes. 
Moreover, further research is needed in order to address issues that have received niver less attention in the literature so far, including the effects of nurse prescribing on the quality of care, provision of information, investigations and referrals, and medication adherence.

\section{CONCLUSION}

Nurses prescribe in comparable ways to physicians and the effects of nurse prescribing on medication and patient outcomes are similar or better when compared to physician prescribing. However, due to methodological weaknesses in this body of research, conclusions must remain tentative. More randomised controlled designs in the field of nurse prescribing are required to enable definitive conclusions about the effects of nurse prescribing.

\section{Author contributions}

JD, SG and MK contributed equally to the study and share joint first authorship. All the authors contributed substantially to the study and approved the final version. PM, JD and SG developed the search strategy. JD and SG carried out the literature search. JD and SG extracted all study data and MK checked all the data extracted. JD, SG and MK wrote the first draft of the paper and PM and AF contributed to subsequent drafts.

\section{Conflict of interest}

No conflicts of interest have been reported.

\section{Funding}

This study was funded by a grant to NIVEL from the Dutch Ministry of Education, Culture and Science.

\section{Ethical approval}

Not required.

\section{Acknowledgements}

This is an update of Van Ruth L, Francke AL, Mistiaen P: Effects of nurse prescribing of medication: a systematic review. Internet Journal of Healthcare Administration 2008, 5: http://dx.doi.org/10.5580/11e, URL: http://ispub.- com/IJHCA/5/2/3311. The review authors acknowledge and thank Lotti van Ruth who was lead author of the original review. She gave permission to update the review and use the original data in the update. She has read and approved the final version of this update.

\section{APPENDiX A. SUPPLEMENTARY DATA}

Supplementary data associated with this article can be found, in the online version, at http://dx.doi.org/10.1016/ j.ijnurstu.2013.12.003 . 
Gielen, S.C., Dekker, J., Francke, A.L., Mistiaen, P., Kroezen, M. The effects of nurse prescribing: a systematic review. International Journal of Nursing Studies: 2014, 51(7), 1048 1061

\section{REFERENCES}

Aarts, J., Koppel, R., 2009. Implementation of computerized physician order entry in seven countries. Health Aff. 28, 404-414.

An Bord Altranais and the National Council for the Professional Devel- opment of Nursing and Midwifery, 2005. Review of Nurses and Midwives in the Prescribing and Administration of Medicinal Products; Final Report. Dublin.

Avery, A., Pringle, M., 2005. Extended prescribing by UK nurses and pharmacists. Br. Med. J. 331, 1154-1155.

Ball, J., 2009. Implementing Nurse Prescribing: An Updated Review of Current Practice Internationally. International Council of Nurses, Geneve.

Banning, M., 2004. Nurse prescribing, nurse education and related research in the United Kingdom: a review of the literature. Nurse Educ. Today 24, 420-427.

Batey, M.V., Holland, J.M., 1985. Prescribing practices among nurse practitioners in adult and family health. Am. J. Public Health 75 (3) 258-262.

Bhanbhro, S., Drennan, V., Grant, R., Harris, R., 2011. Assessing the contribution of prescribing in primary care by nurses and profes- sionals allied to medicine: a systematic review of literature. BMC Health Serv. Res. 11, 330.

Black, A., 2012. Non-medical prescribing by nurse practitioners in acci- dent \& emergency and sexual health: a comparative study. J. Adv. Nurs. 69, 535-545.

Bradley, E., Nolan, P., 2007. Impact of nurse prescribing: a qualitative study. J. Adv. Nurs. 59, 120-128.

Buchan, J., Calman, L., 2004. Implementing Nurse Prescribing. An Updated Review of Current Practice Internationally. International Council of Nurses.

Butler, C.C., Rees, M., Kinnersley, P., Rollnick, S., Hood, K., 2001. A case study of nurse management of upper respiratory tract infections in general practice. J. Adv. Nurs. 33 (3) 328-333.

Cipher, D.J., Hooker, R.S., Guerra, P., 2006. Prescribing trends by nurse practitioners and physician assistants in the United States. J. Am. Acad. Nurse Pract. 18, 291-296.

Clarke, M., Oxman, A.D., 2003. Cochrane Reviewer's Handbook 4.1.6. Oxford.

Cochrane Effective Practice and Organisation of Care Review Group, 2002.

The Data Collection Checklist. Institute of Population Health, Uni- versity of Ottawa, Ottawa, Canada.

Cox, C., Jones, M., 2000. An evaluation of the management of patients with sore throats by practice nurses and GPs. Br. J. Gen. Pract. 50 (460) 872-876.

Creedon, R., O'Connell, E., McCarthy, G., Lehane, B., 2009. An evaluation of nurse prescribing. Part 1: a literature review. Br. J. Nurs. 18, 1322-1327.

Crown, J., Miller, P., 2005. Access to the whole BNF: a long-awaited triumph or the wrong decision? British National Formulary. Nurse Prescrib. 3, 228-230.

Davis, K., Drennan, V., 2007. Evaluating nurse prescribing behaviour using constipation as a case study. Int. J. Nurs. Pract. 13, 243-253.

Department of Health, 1999. Review of Prescribing, Supply \& Adminis- tration of Medicines: Final Report. London.

Department of Health, 2002. Extending Independent Nurse Prescribing within the NHS in England: A Guide for Implementation. Department of Health, London.

Department of Health, 2010a. Nurse Prescribing FAQ. http://webarchive.nationalarchives.gov.uk/+/www.dh.gov.uk/en/Healthcare/Medicinespharmacyandindustry/Prescriptions/TheNon-MedicalPrescribingProgramme/Nurseprescribing/DH_4123003.

Department of Health, 2010b. Supplementary Prescribing FAQ. http:// webarchive.nationalarchives.gov.uk/+/www.dh.gov.uk/en/Healthcare/Medicinespharmacyandindustry/Prescriptions/TheNon-MedicalPrescribingProgramme/Supplementaryprescribing/DH_4123034.

Department of Health, 2010c. Patient Group Directions. http:// www.dh.gov.uk/en/Publicationsandstatistics/Publications/PublicationsPolicyAndGuidance/Browsable/DH_4898318.

Drennan, J., Naughton, C., Allen, D., Hyde, A., Felle, P., et al., 2009. National Independent Evaluation of the Nurse and Midwife Prescribing Initia- tive. University College Dublin, Dublin. 
Gielen, S.C., Dekker, J., Francke, A.L., Mistiaen, P., Kroezen, M. The effects of nurse prescribing: a systematic review. International Journal of Nursing Studies: 2014, 51(7), 1048 1061

Dutch House of Representatives, 2011. Tweede Kamer der Staten-Gen- eraal, vergaderjaar 2010-2011, 32 196, 32 261, nr. 13, Brief van de Minister van Volksgezondheid, Welzijn en Sport [The Dutch House of Representatives, year 2010-2011, 32 196, 32 261, no. 13, Letter of the Minister of Health, Welfare and Sport]. Den Haag.

Einhorn, R.F., Trias, M., 1978. Differences between physicians and nurses in providing family planning services: findings from a Bogota clinic. Stud. Family Plann. 9 (2/3) 35-38.

Emmerton, L., Marriott, J., Bessell, T., Nissen, L., Dean, L., 2005. Pharmacists and prescribing rights: review of international developments. J. Pharm. Pharm. Sci. 8, 217-225.

Feldman, S., Bachman, J., Cuffel, B., Friesen, B., McCabe, J., 2003. Advanced practice psychiatric nurses as a treatment resource: survey and analysis. Adm. Policy Ment. Health 30 (6) 479-492.

Ferguson, B., Luker, K., Smith, K., Austin, L., Hogg, C., 1998. Preliminary findings from an economic analysis of nurse prescribing. Int. J. Pharm. Pract. 6 (3) 3-132. Fisher, S.E., Vaughan-Cole, B., 2003. Similarities and differences in clients treated and in medications prescribed by APRNs and psychiatrists in a CMHC. Arch. Psychiatr. Nurs. 17 (3) 101-107.

Fletcher, C.E., Copeland, L.A., Lowery, J.C., Reeves, P.J., 2011. Nurse practitioners as primary care providers within the VA. Mil. Med. 176, 791-797.

Foreman, D.M., Morton, S., 2011. Nurse-delivered and doctor-delivered care in an attention deficit hyperactivity disorder follow-up clinic: a comparative study using propensity score matching. J. Adv. Nurs. 67, 1341-1348.

Gambino, K.K., Planavsky, L., Gaudette, H., 2009. Transition toward a nurse practitionermanaged clinic. J. Cardiovasc. Nurs. 24, 132-139. Hansen, T., Skjeldestad, F.E., 2007. Adolescents: is there an association between knowledge of oral contraceptives and profession of provider? Eur. J. Contracept. Reprod. Health Care 12, 303-308. Hartley, J., 2003. Nurse prescribing the big picture. Nurs. Times 99, 22-25.

Harris, J., Taylor, J., Mackie, C., 2004. Research Literature Review on Prescribing. Scottish Executive Social Research.

Higgins, J.P.T., Green, S., 2005. Cochrane Handbook for Systematic Reviews of Interventions 4.2.5. John Wiley \& Sons, Chichester, UK.

Higgins, J.P.T., Green, S., 2011. Cochrane Handbook for Systematic Reviews of Interventions 5.1.0. John Wiley \& Sons Ltd, Chichester UK.

Hooker, R.S., Cipher, D.J., 2005. Physician assisant and nurse practitioner prescribing: 1997-2002. J. Rural Health 21 (4) 355-360.

Horrocks, S., Anderson, E., Salisbury, C., 2002. Systematic review of whether nurse practitioners working in primary care can provide equivalent care to doctors. $\mathrm{Br}$. Med. J. 324, 819-823.

Horton, R., 2002. Nurse-prescribing in the UK: right but also wrong. Lancet 359 (9321) 1875-1876.

Houweling, S.T., Kleefstra, N., Groenier, K.H., Meyboom-de Jong, B., Bilo, H.J.G., 2005a. De diabetesverpleegkundige als hoofdbehandelaar bij patienten met diabetes mellitus type 2 in de tweede lijn: een ger- andomiseerd onderzoek. Taakdelegatie in de eerste- en tweedelijns diabeteszorg: resultaten van de DISCOURSE-studies. Rijksuniversiteit Groningen, Groningen, pp. 69-86.

Houweling, S.T., Kleefstra, N., Groenier, K.H., Meyboom-de Jong, B., Bilo, H.J.G., 2005b. De diabetesverpleegkundige als hoofdbehandelaar bij patienten met diabetes mellitus type 2 in de tweede lijn: de inspan- ningen en de kosten. Taakdelegatie in de eerste- en tweedelijns diabeteszorg: resultaten van de DISCOURSE-studies. Rijksuniversiteit Groningen, Groningen, pp. 87-96.

Houweling, S.T., Kleefstra, N., Groenier, K.H., Meyboom-de Jong, B., Bilo, H.J.G., 2005c. De praktijkondersteuner als hoofdbehandelaar bij patienten met diabetes mellitus type 2 in de eerste lijn: een geran- domiseerd onderzoek. Taakdelegatie in de eerste- en tweedelijns diabeteszorg: resultaten van de DISCOURSE-studies. Rijksuniversiteit Groningen, Groningen, pp. 49-68.

Houweling, S.T., Kleefstra, N., VanBallegooie, E., Verhoeven, S., Bilo, H.J.G., et al., 2006. Taakverschuivingen in de eerstelijns diabeteszorg: een enquete onder ruim vierhonderd praktijkondersteuners. Huisarts \& Wetensch 49 (3) 118-122. 
Gielen, S.C., Dekker, J., Francke, A.L., Mistiaen, P., Kroezen, M. The effects of nurse prescribing: a systematic review. International Journal of Nursing Studies: 2014, 51(7), 1048 1061

Houweling, S.T., Kleefstra, N., Van Hateren, K.J.J., Kooy, A., Groenier, K.H., et al., 2009. Diabetes specialist nurse as main care provider for patients with type 2 diabetes. Neth. J. Med. 67, 279-284.

Jacobs, J.T., 2005. Treatment of depressive disorders in split versus integrated therapy and comparisons of prescriptive practices of psy- chiatrists and advanced practice registered nurses. Arch. Psychiatr. Nurs. 19 (6) 256-263.

James, J., 2004. Supplementary prescribing by a diabetes specialist nurse on a hospital ward. Nurs. Prescrib. 2 (3) 112-116.

Jones, A., 2009. Nurse Prescribing in Mental Health. Wiley-Blackwell, Chichester.

Jones, K., Edwards, M., While, A., 2011. Nurse prescribing roles in acute care: an evaluative case study. J. Adv. Nurs. 67, 117-126.

Kinnersley, P., Anderson, E., Parry, K., Clement, J., Archard, L., et al., 2000. Randomised controlled trial of nurse practitioner versus general practitioner care for patients requesting same day consultations in primary care. BMJ 320 (7241) 1043-1048.

Kroezen, M., van Dijk, L., Groenewegen, P.P., Francke, A.L., 2011. Nurse prescribing of medicines in Western European and Anglo-Saxon countries: a systematic review of the literature. BMC Health Serv. Res. 11 (1) 127, http://dx.doi.org/10.1186/1472-6963-11-127.

Kroezen, M., Francke, A.L., Groenewegen, P.P., Van Dijk, L., 2012. Nurse prescribing of medicines in Western European and Anglo-Saxon countries: a survey on forces, conditions and jurisdictional control. Int. J. Nurs. Stud. 49, 1002-1012.

Kuethe, M., Vaessen, V.A., Mulder, P., Bindels, P., van, A.W., 2011. Paediatric asthma outpatient care by asthma nurse, paediatrician or general practitioner: randomised controlled trial with two-year fol- low-up. Prim. Care Respir. J. 20, 84-91.

Ladd, E., 2005. The use of antibiotics for viral upper respiratory tract infections: an analysis of nurse practitioner and physician prescribing practices in ambulatory care. J. Am. Acad. Nurse Pract. 17 (10) 416-424.

Landis, J.R., Koch, C.G., 1977. The measurement of observer agreement for categorical data. Biometrics 33, 159-174.

Latter, S., Courtenay, M., 2004. Effectiveness of nurse prescribing: a review of the literature. J. Clin. Nurs. 13 (1) 26-32.

Latter, S., Maben, J., Myall, M., Courtenay, M., Young, A., et al., 2005. An evaluation of Extended Formulary Independent Nurse Prescribing; Executive Summary of Final Report. Department of Health.

Mahoney, D.F., 1994. Appropriateness of geriatric prescribing decisions made by nurse practitioners and physicians. Image J. Nurs. Sch. 26 (1) 41-46.

Mallett, J., Guerrero, D., Rhys-Evans, F., Faithfull, S., 1997. Nurse prescrib- ing by protocol - nurse-run clinics ensure patients get their pills on time. Nurs. Times 93 (8) 50-53.

Ministry of Health WaS, 2011. Besluit van 21 december 2011. houdende tijdelijke regels inzake de zelfstandige bevoegdheid tot het verrichten van voorbehouden handelingen van verpleegkundig specialisten (Tij- delijk besluit zelfstandige bevoegdheid verpleegkundig specialisten) [Decision of 21 December, on temporary rules relating to the auton- omous power to perform restricted actions of nurse specialists (Temporary autonomous decision power nurse specialists)]. Staats- blad van het Koninkrijk der Nederlanden 659.

Moher, D., Liberati, A., Tetzlaff, J., Altman, D.G., 2009. Preferred Reporting Items for Systematic Reviews and Meta-Analyses: The PRISMA State- ment. PLoS Med. 6 (7) e1000097.

National Nursing and Nursing Education Taskforce, 2006. National Nurse Prescribing Glossary. National Nursing \& Nursing Education Task- force, Melbourne. Nilsson, M., 1994. Opposition to nurse-prescribing in Sweden. Lancet 1077.

O'Connell, E., Creedon, R., McCarthy, G., Lehane, B., 2009. An evaluation of nurse prescribing. Part 2: a literature review. Br. J. Nurs. 18, 1398-1402.

Pharmaceutisch Weekblad, 2011. Finland: voorschrijvende verpleegkun- digen [Finland: Prescribing Nurses]. Available from: http://www pw nl/nieuws/nieuwsberichten/finlandvoorschrijvende-verpleegkundi- gen (accessed 2401 2011).

Pritchard, A., Kendrick, D., 2001. Practice nurse and health visitor management of acute minor illness in a general practice. J. Adv. Nurs. 36 (4) 556-562. 
Gielen, S.C., Dekker, J., Francke, A.L., Mistiaen, P., Kroezen, M. The effects of nurse prescribing: a systematic review. International Journal of Nursing Studies: 2014, 51(7), 1048 1061

Raad voor de Volksgezondheid en Zorg, 2002. Taakherschikking in de gezondheidzorg [Task substitution in healthcare]. Zoetermeer. Roumie, C.L., Halasa, N.B., Edwards, K.M., Zhu, Y., Dittus, R.S., et al., 2005.

Differences in antibiotic prescribing among physicians, residents, and nonphysician clinicians. Am. J. Med. 118 (6) 641-648.

Royal College of Nursing, 2004. Patient Group Directions: Guidance and Information for Nurses. Royal College of Nursing, London.

Running, A., Kipp, C., Mercer, V., 2006. Prescriptive patterns of nurse practitioners and physicians. J. Am. Acad. Nurse Pract. 18, 228-233.

Ryan, T., Cash, K., Hannis, D., 1999. Nurse prescribing and in-patient alcohol detoxification. J. Subst. Use 4 (4) 133-141.

Sandø, A.D., Aanes, S.G., Slordal, L., Spigset, O., 2010. Routines for first prescription or oral contraceptives. Tidsskr Nor Laegeforen 130, 2344-2348.

Shum, C., Humphreys, A., Wheeler, D., Cochrane, M.A., Skoda, S., et al., 2000. Nurse management of patients with minor illnesses in general practice: multicentre, randomised controlled trial. BMJ 320 (7241) 1038-1043.

Siriwardena, A.N., 2006. The rise and rise of non-medical prescribing. Q. Prim. Care 14, 13.

Spitzer, W.O., Sackett, D.L., Sibley, J.C., Roberts, R.S., Gent, M., et al., 1974. The burlington randomized trial of the nurse practitioner. N. Engl. J. Med. 290, 251-256.

Tobe, S.W., Pylypchuk, G., Wentworth, J., Kiss, A., Szalai, J.P., et al., 2006. Effect of nursedirected hypertension treatment among First Nations people with existing hypertension and diabetes mellitus: the Dia- betes Risk Evaluation and Microalbuminuria (DREAM 3) randomized controlled trial. Can. Med. Assoc. J. 174, 1267-1271.

Undeland, D.K., Kowalski, T.J., Berth, W.L., Gundrum, J.D., 2010. Appro- priately prescribing antibiotics for patients with pharyngitis: a phy- sician-based approach vs a nurse-only triage and treatment algorithm. Mayo Clin. Proc. 85, 1011-1015.

Van Ruth, L., Francke, A.L., Mistiaen, P., 2008. Effects of nurse prescribing of medication: a systematic review. Int. J. Healthc. Admin. 5 , http:// dx.doi.org/10.5580/11e.

Venning, P., Durie, A., Roland, M., Roberts, C., Leese, B., 2000. Randomised controlled trial comparing cost effectiveness of general practitioners and nurse practitioners in primary care. BMJ 320 (7241) 1048-1053.

Watterson, A., Turner, F., Coull, A., Murray, I., 2009. An Evaluation of the Expansion of Nurse Prescribing in Scotland. Scottish Government Social Research, Edinburgh.

Williams, K., Kukla, M., Bond, G.R., McKasson, M., Salyers, M.P., 2009. Can a nurse practitioner serve in the prescriber role on an assertive com- munity treatment team? Am. J. Psychiatr. Rehabil. 12, 205-224.

Wilhelmsson, S., Foldevi, M., 2003. Exploring views on Swedish district nurses' prescribing a focus group study in primary health care. J. Clin. Nurs. 12, 643-650. 
Gielen, S.C., Dekker, J., Francke, A.L., Mistiaen, P., Kroezen, M. The effects of nurse prescribing: a systematic review. International Journal of Nursing Studies: 2014, 51(7), 1048 1061

\title{
FIGURES AND TABLES
}

\section{Box 1. General models of nurse prescribing}

\begin{abstract}
Independent prescribing
Legally permitted and qualified independent prescribers are responsible for the clinical assessment of a patient, the establishment of a diagnosis and decisions about the appropriateness of medication, treatment or an appliance, including the issuing of a prescription (Department of Health, 2010a; Watterson et al., 2009). Prescribing usually takes place from a limited formulary - a list containing a limited and defined number of medicines that can be prescribed - or an open formulary. This form of prescribing is also referred to as initial, autonomous, substitutive or open prescribing (National Nursing and Nursing Education Taskforce, 2006; Van Ruth et al., 2008)
\end{abstract}

Supplementary prescribing

Supplementary prescribing is defined as a voluntary partnership between an independent prescriber - a doctor or a dentist - and a supplementary prescriber - usually a nurse or a pharmacist. After the initial assessment and diagnosis of a patient's condition have been carried out by the independent prescriber, the nurse may prescribe from an open or limited formulary and will collaborate or consult with the independent prescriber before issuing the prescription, even though direct supervision is not required (Department of Health, 2010b; National Nursing and Nursing Education Taskforce, 2006; Watterson et al., 2009)

In the United Kingdom, an important additional feature of supplementary prescribing is the collaboration between the independent and supplementary prescribers in drawing up a Clinical Management Plan which needs to be approved by the patient before implementation (Department of Health, $2010 \mathrm{~b}$; Hartley, 2003). Supplementary prescribing is also known as dependent, collaborative, semi-autonomous or complementary prescribing (National Nursing and Nursing Education Taskforce, 2006; Van Ruth et al., 2008)

Patient group directions

Patient group directions (PGDs), formerly known as group protocols, refer to written instructions for the supply and administration of named medicines in an identified clinical situation (Department of Health, 2010c; National Nursing and Nursing Education Taskforce, 2006; Royal College of Nursing, 2004; Van Ruth et al., 2008). Drawn up by a multidisciplinary team, they are specifically designed for a particular group of patients with a specific condition, thus excluding individualised prescriptions (Harris et al., 2004). Group protocols should not be seen as independent prescribing, since nurses or other health-care professionals are only allowed to supply and administer medications within the strict terms of a predetermined protocol, albeit using their own assessment of patient needs (Hartley, 2003; Royal College of Nursing, 2004) 
Gielen, S.C., Dekker, J., Francke, A.L., Mistiaen, P., Kroezen, M. The effects of nurse prescribing: a systematic review. International Journal of Nursing Studies: 2014, 51(7), 10481061

FIG. 1. FLOW DIAGRAM OF THE INCLUSION PROCESS.

\begin{tabular}{|c|c|c|c|c|c|c|}
\hline \multicolumn{7}{|c|}{ Database searches, number of hits } \\
\hline BIOMED & CINAHL & CDSR & CCT & EMBASE & INVERT & NIVELCAT \\
\hline 85 & 1791 & 582 & 99 & 3298 & 39 & 11 \\
\hline PICARTA & PUBMED & SCHOLAR & SCI & VHINL & WEBSITES & \\
\hline 410 & 1699 & 306 & 1800 & 549 & 27 & \\
\hline
\end{tabular}

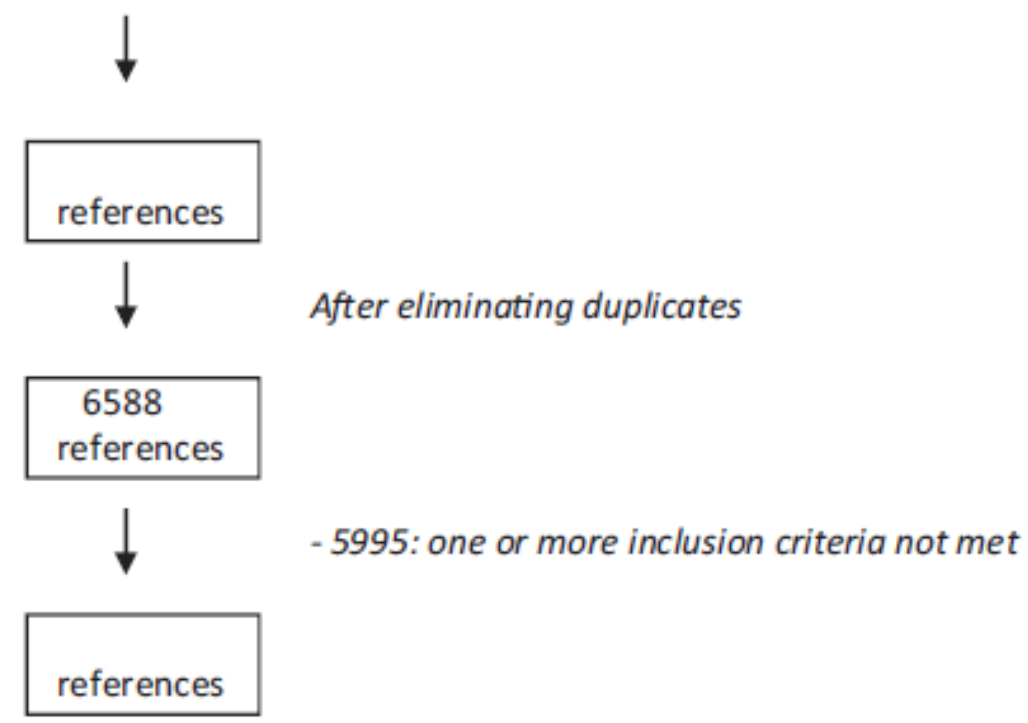

593 references, 578 obtained, 15 not obtained

FULL TEXT

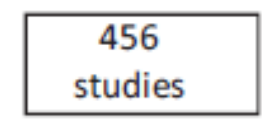

- 562 studies excluded because:

- $\quad$ no empirical research $(N=218)$

- reviews $(N=14)$

- not about nurse prescribing $(N=40)$

- no comparative design $(N=258)$

- not about effects of nurse prescribing $(\mathrm{N}=25)$

- not about quantity or patient outcomes $(N=7)$

studies

PREVIOUS REVIEW

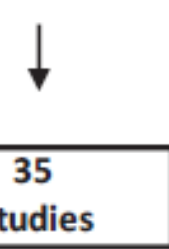

+21 studies included that met inclusion criteria

FINAL SET 
Gielen, S.C., Dekker, J., Francke, A.L., Mistiaen, P., Kroezen, M. The effects of nurse prescribing: a systematic review. International Journal of Nursing Studies: 2014, 51(7), 10481061

Table 1

Total amount of medication prescribed by nurses compared with physicians.

\begin{tabular}{|c|c|c|c|c|c|}
\hline \multirow[t]{2}{*}{ Study } & \multirow[t]{2}{*}{ Country } & \multirow[t]{2}{*}{ Patient group } & \multicolumn{3}{|c|}{$\begin{array}{l}\text { Total amount of medication prescribed by } \\
\text { nurses as compared to physicians }\end{array}$} \\
\hline & & & Less & Same & More \\
\hline Houweling et al. (2009) & The Netherlands & Diabetes & $\mathrm{X}^{\mathrm{a}}$ & & \\
\hline Fletcher et al. (2011) & USA & Hypertension/diabetes & $\mathrm{X}^{\mathrm{b}}$ & $\mathrm{x}$ & \\
\hline Fisher \& Vaughan-Cole (2003) & USA & Schizophrenia or depression & Unclear & $\mathrm{x}$ & \\
\hline Houweling et al. (2005a) & The Netherlands & Diabetes & $\mathrm{X}^{\mathrm{a}}$ & $\mathrm{x}$ & \\
\hline Ferguson et al. (1998) & UK & Various & & $\mathrm{x}$ & \\
\hline Sandø et al. (2010) & Norway & $\begin{array}{l}\text { Patients who received oral } \\
\text { contraceptives }\end{array}$ & & $\mathrm{x}$ & \\
\hline Cipher et al. (2006) & USA & Various & & $\mathrm{x}$ & $X^{c}$ \\
\hline Running et al. (2006) & USA & Various & $X^{d}$ & $\mathrm{x}$ & $\mathrm{X}^{\mathrm{e}}$ \\
\hline Jacobs (2005) & USA & $\begin{array}{l}\text { Depression, dysthymia or } \\
\text { bipolar II disorders }\end{array}$ & Unclear & Unclear & Unclear \\
\hline
\end{tabular}

\footnotetext{
a For cholesterol-lowering agents.

b For cardiovascular agents.

c In non-metropolitan areas.

${ }^{d}$ For bronchodilators.

e For decongestants for bronchitis.
}

\section{Table 2}

Number of patients prescribed medication by nurses versus physicians.

\begin{tabular}{|c|c|c|c|c|c|}
\hline \multirow[t]{2}{*}{ Study } & \multirow[t]{2}{*}{ Country } & \multirow[t]{2}{*}{ Patient group } & \multicolumn{3}{|c|}{$\begin{array}{l}\text { Number of patients prescribed by nurse as } \\
\text { compared to physician/psychiatrist }\end{array}$} \\
\hline & & & Fewer & Same & More \\
\hline Cipher et al. (2006) & USA & Various & & $\mathrm{x}$ & \\
\hline Cox and Jones (2000) & UK & Sore throats & & $\mathrm{x}$ & \\
\hline Feldman et al. (2003) & USA & Mental health patients & & $\mathrm{x}$ & \\
\hline Jones et al. (2011) & UK & Hypertension/renal problems & & $\mathrm{x}$ & \\
\hline Ladd (2005) & USA & URTI & & $\mathrm{x}$ & \\
\hline Pritchard \& Kendrick (2001) & UK & Acute minor illnesses & & $\mathrm{X}$ & \\
\hline Shum et al. (2000) & UK & Various & & $\mathrm{X}$ & \\
\hline Venning et al. (2000) & UK & Various & & $\mathrm{x}$ & \\
\hline Hooker and Cipher (2005) & USA & Various & & $\mathrm{x}$ & $\mathrm{X}^{\mathrm{a}}$ \\
\hline Roumie et al. (2005) & USA & Various & & & $\mathrm{x}$ \\
\hline
\end{tabular}

\footnotetext{
${ }^{a}$ Only in rural areas.
} 
Gielen, S.C., Dekker, J., Francke, A.L., Mistiaen, P., Kroezen, M. The effects of nurse prescribing: a systematic review. International Journal of Nursing Studies: 2014, 51(7), 1048 1061

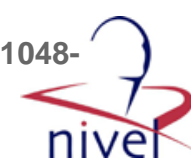

FIG. 2. NUMBER OF PATIENTS BEING PRESCRIBED MEDICATION IN PRIMARY CARE IN THE UK.

Revien. nusepesterting

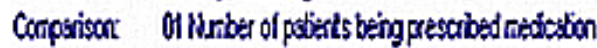

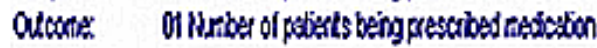

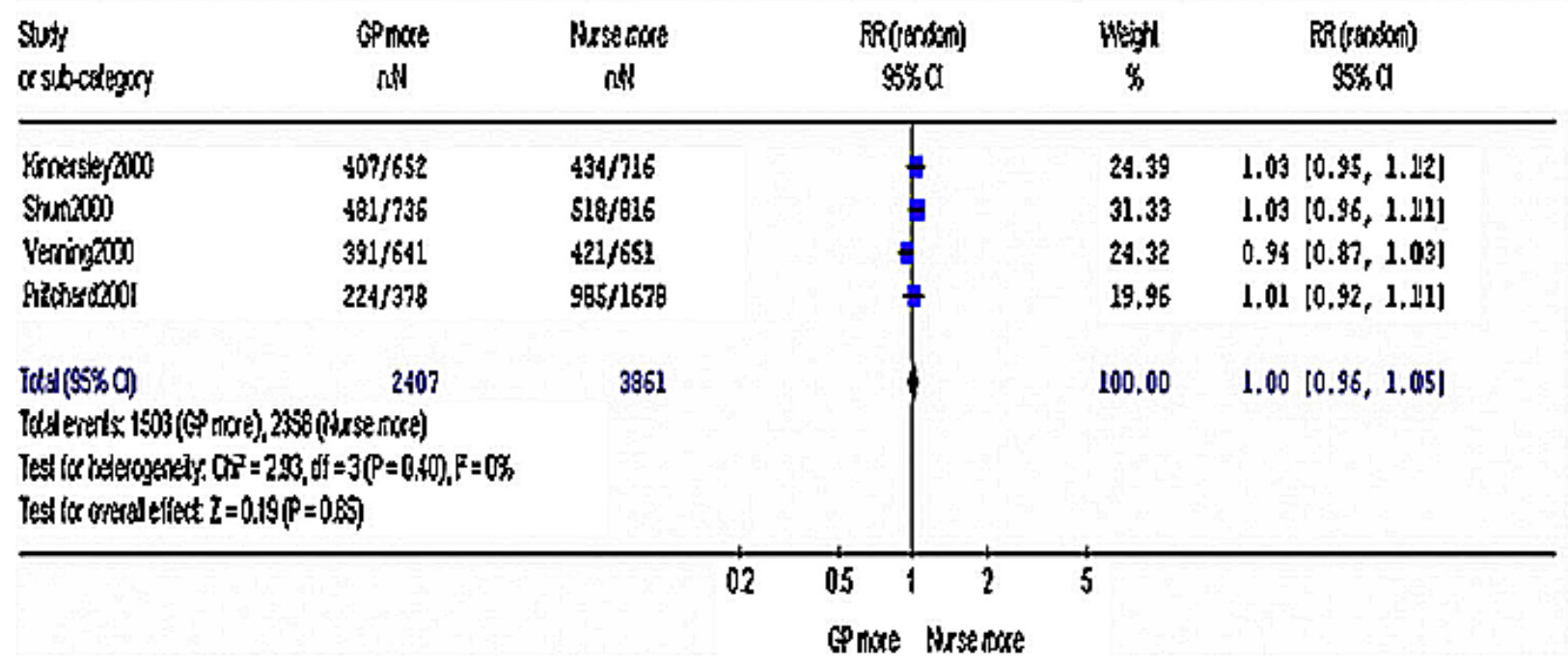

Table 3

Clinical outcomes of patients receiving prescriptions from nurses versus physicians.

\begin{tabular}{|c|c|c|c|c|c|}
\hline \multirow[t]{2}{*}{ Study } & \multirow[t]{2}{*}{ Country } & \multirow[t]{2}{*}{ Type of patient } & \multicolumn{3}{|l|}{ Clinical outcomes } \\
\hline & & & $\begin{array}{l}\text { Better outcomes } \\
\text { for nurses }\end{array}$ & No difference & $\begin{array}{l}\text { Better outcomes } \\
\text { for physicians }\end{array}$ \\
\hline Cox and Jones (2000) & UK & Sore throats & $\mathrm{x}^{\mathrm{b}}$ & $\mathrm{X}^{\mathrm{c}}$ & \\
\hline Einhorn and Trias (1978) & Colombia & Women seeking contraceptives & & $\mathrm{x}$ & \\
\hline Fletcher et al. (2011) & USA & Diabetes and/or hypertension & & $\mathrm{x}$ & \\
\hline Houweling et al. (2005c) & Netherlands & Diabetes & & $\mathrm{x}$ & \\
\hline Shum et al. (2000) & UK & Various & & $\mathrm{x}$ & \\
\hline Spitzer et al. (1974) & Canada & Various & & $\mathrm{x}$ & \\
\hline Venning et al. (2000) & UK & Various & & $\mathrm{x}$ & \\
\hline Houweling et al. (2005b) & The Netherlands & Diabetes & & $\mathrm{x}$ & $X^{d}$ \\
\hline Houweling et al. (2009) & Netherlands & Diabetes & & $\mathrm{X}$ & $\mathrm{X}^{\mathrm{d}}$ \\
\hline James (2004) & $\mathrm{UK}$ & Diabetes & Unclear & Unclear & Unclear \\
\hline
\end{tabular}

${ }^{a}$ For diastolic blood pressure.

${ }^{b}$ For perception of being back to normal health and number of days for sore throat to settle.

${ }^{c}$ For number of patients whose sore throats had settled.

${ }^{\mathrm{d}}$ For cholesterol/HDL ratio. 
Gielen, S.C., Dekker, J., Francke, A.L., Mistiaen, P., Kroezen, M. The effects of nurse prescribing: a systematic review. International Journal of Nursing Studies: 2014, 51(7), 10481061

Table 4

Patients' satisfaction with care provided by nurses versus physicians.

\begin{tabular}{|c|c|c|c|c|c|}
\hline \multirow[t]{2}{*}{ Study } & \multirow[t]{2}{*}{ Country } & \multirow[t]{2}{*}{ Patient group } & \multicolumn{3}{|c|}{$\begin{array}{l}\text { Patient satisfaction with care provided } \\
\text { by nurses versus physicians }\end{array}$} \\
\hline & & & Less & Same & Greater \\
\hline Williams et al. (2009) & USA & Mental illness & $\mathrm{X}$ & & \\
\hline Kinnersley et al. (2000) & UK & Various & & $\mathrm{X}$ & $\mathrm{X}^{\mathrm{a}}$ \\
\hline Cox and Jones (2000) & UK & Sore throats & & $\mathrm{X}$ & \\
\hline Foreman and Morton (2011) & UK & ADHD & & $\mathrm{x}$ & \\
\hline Pritchard and Kendrick (2001) & UK & Various & & $\mathrm{x}$ & \\
\hline Spitzer et al. (1974) & Canada & Various & & $\mathrm{x}$ & \\
\hline Gambino et al. (2009) & USA & Cardiology and rehabilitation & & & $\mathrm{X}$ \\
\hline Houweling et al. (2005b) & Netherlands & Diabetes & & & $\mathrm{X}$ \\
\hline Houweling et al. (2005c) & Netherlands & Diabetes & & & $\mathrm{X}$ \\
\hline Houweling et al. (2009) & Netherlands & Diabetes & & & $\mathrm{X}$ \\
\hline Jones et al. (2011) & UK & Hypertension and/or renal & & & $\mathrm{X}$ \\
\hline Shum et al. (2000) & UK & Various & & & $\mathrm{X}$ \\
\hline Venning et al. (2000) & UK & Various & & & $\mathrm{X}$ \\
\hline
\end{tabular}

a Children are more satisfied. 